\title{
Detection of phosphatidylserine-positive exosomes for the diagnosis of early-stage malignancies
}

\author{
Raghava Sharma ${ }^{1}$, Xianming Huang ${ }^{1}$, Rolf A Brekken ${ }^{1,2}$ and Alan J Schroit ${ }^{\star, 3,4}$ \\ ${ }^{1}$ Hamon Center for Therapeutic Oncology Research, UT Southwestern Medical Center, Dallas, TX 75390, USA; ${ }^{2}$ Department of \\ Surgery, UT Southwestern Medical Center, Dallas, TX 75390, USA; ${ }^{3}$ Harold Simmons Comprehensive Cancer Center, UT \\ Southwestern Medical Center, Dallas, TX 75390, USA and ${ }^{4}$ Department of Immunology, UT Southwestern Medical Center, Dallas \\ 75390, TX, USA
}

Background: There has been increasing interest in the detection of tumour exosomes in blood for cancer diagnostics. Most studies have focussed on miRNA and protein signatures that are surrogate markers for specific tumour types. Because tumour cells and tumour-derived exosomes display phosphatidylserine (PS) in their outer membrane leaflet, we developed a highly sensitive ELISA-based system that detects picogram amounts of exosomal phospholipid in plasma as a cancer biomarker.

Methods: This report describes the development of a highly specific and sensitive ELISA for the capture of PS-expressing tumour exosomes in the blood of tumour-bearing mice. To monitor the relationship between tumour burden and tumour exosome plasma concentrations, plasma from one transplantable breast cancer model (MDA-MB-231) and three genetic mouse models (MMTV-PyMT; breast and KIC and KPC; pancreatic) were screened for captured exosomal phospholipid.

Results: We show that quantitative assessment of PS-expressing tumour exosomes detected very early-stage malignancies before clinical evidence of disease in all four model systems. Tumour exosome levels showed significant increases by day 7 after tumour implantation in the MDA-MB-231 model while palpable tumours appeared only after day 27. For the MMTV-PyMT and KIC models, tumour exosome levels increased significantly by day $49(P \leqslant 0.0002)$ and day $21(P \leqslant 0.001)$ while tumours developed only after days 60 and 40, respectively. For the KPC model, a significant increase in blood exosome levels was detected by day 70 $(P=0.023)$ when only preinvasive lesions are microscopically detectable.

Conclusions: These data indicate that blood PS exosome levels is a specific indicator of cancer and suggest that blood PS is a biomarker for early-stage malignancies.

There is increasing evidence that, in contrast to normal nontumourigenic cells, many tumour cells expose PS on their surface (Connor et al, 1989; Utsugi et al, 1991; Riedl et al, 2011; Bujak et al, 2015; Vallabhapurapu et al, 2015) by a mechanism that is unrelated to PS externalisation commonly seen in dying apoptotic cells (Dong et al, 2009; Vallabhapurapu et al, 2015). Because tumour cells are known to secrete large amounts of plasma membranederived exosomes to the bloodstream (Keller et al, 2009; Logozzi et al, 2009; Rabinowits et al, 2009; Khan et al, 2012; Noerholm et al, 2012; Szajnik et al, 2013; Melo et al, 2015; Cappello et al, 2017), we tested whether the presence of PS-expressing exosomes in blood would be diagnostic for cancer. For this purpose, we developed a highly stringent ELISA for the detection of PS in patient blood. Our data showed that cancer patient blood, but not blood from tumour-free individuals, contained significant amounts of tumour-derived PS-expressing exosomes, the presence of which was diagnostic for cancer (Lea et al, 2017). Although this retrospective study predicted with $100 \%$ accuracy, the presence

*Correspondence: Dr AJ Schroit; E-mail: alan.schroit@utsouthwestern.edu

Revised 25 May 2017; accepted 26 May 2017; published online 22 June 2017

(C) 2017 Cancer Research UK. All rights reserved 0007-0920/17 
of disease in patients with suspect ovarian malignancies, the test would need to be significantly more sensitive for the detection of indolent disease in asymptomatic patients.

The stringency of the PS ELISA was ensured by employing a binary PS-dependent test system consisting of beta-2-glycoprotein 1 ( $\beta 2 \mathrm{GP} 1)$-mediated PS-specific exosome capture (Wurm, 1984; Willems et al, 1996) with a tetravalent $\beta 2 \mathrm{GP} 1$ antibody and detection with PS-specific annexin 5. However, maximum test sensitivity was likely compromised because (1) annexin 5 requires 1-2 mM calcium for PS binding while $\mathrm{Ca}^{2+}$ is known to impede the binding of $\beta 2 \mathrm{GP} 1$ to PS (Wurm, 1984; Willems et al, 1996), (2) phospholipids are highly mobile in the plane of bilayer membranes raising the possibility that a large fraction of the exosomal PS could have been sequestered to the $\beta 2 \mathrm{GP} 1 /$ antibody contact points precluding detection with annexin 5 and (3) individual patient levels of $\beta 2 \mathrm{GP} 1$ could have a significant impact on particle capture to the antibody-coated ELISA plate.

In principle, these issues could be overcome by employing (1) a $\mathrm{Ca}^{2+}$-independent assay system, (2) noncompeting exosome capture and detection technologies and (3) a capture antibody that is not dependent on patient $\beta 2 \mathrm{GP} 1$ levels. Using this rationale for modification of the detection system, we show that a unary system was of sufficient stringency to distinguish between normaland tumour-derived blood exosomes with $>25$-fold increase in sensitivity over previously described methodologies (Lea et al, 2017). Here we show the utility of this system using four mouse tumour models where, without exception, tumours were detected much earlier than was clinical evidence of disease. Taken together, these data show that the presence of exosomal PS in blood is a reliable surrogate for the detection of early malignancies.

\section{MATERIALS AND METHODS}

Antibodies: expression of an engineered bivalent 'betabody'. Betabody was generated by fusing domains 1 and 5 of the PSbinding plasma protein, human $\beta 2 \mathrm{GP}$, to the C-termini of the $\mathrm{CH} 3$ domains of the Fc fragment of human IgG1. A Gly4Ser linker was inserted between the $\mathrm{CH} 3$ domains, domains 1 and 5 of $\beta 2 \mathrm{GP} 1$. The recombinant betabody was expressed as a dimer. The fusion protein was expressed by transfection of the plasmids in $\mathrm{CHO}$ cells. Expressing clones were identified by PS ELISA and stably transfected clones were selected. Fusion proteins were purified from culture supernatants with protein A-Sepharose chromatography, dialysed into PBS and stored at $-70{ }^{\circ} \mathrm{C}$.

Mouse samples. Longitudinal blood samples $(\sim 150 \mu \mathrm{l})$ were collected by submandibular bleeds (Golde et al, 2005) at the indicated time points. Blood was collected in $1.5 \mathrm{ml}$ polypropylene tubes containing $\mathrm{K}_{3}$ EDTA $(\sim 0.1 \mathrm{mg})$. Platelet poor plasma was prepared by centrifugation for $10 \mathrm{~min}$ at $700 \mathrm{~g}$ to remove blood cells. Plasma was collected, centrifuged again at $12000 \mathrm{~g}$ for $5 \mathrm{~min}$ to remove platelets and large extracellular vesicles and stored at $-20^{\circ} \mathrm{C}$.

Patient blood. Blood was collected from patients with confirmed ovarian malignancies and from healthy sex-matched donors obtained from the UT Southwestern Gynecologic Oncology clinics and UT Southwestern Biomarker Research Core, respectively. All samples were collected in accordance with UT Southwestern Institutional Review Board (STU 062010-201 and STU 092014013). Donors were selected at random without any inclusion or exclusion criteria. Informed consent was obtained before blood collection. Blood was collected in $\mathrm{K}_{3}$ EDTA vacutainers. Platelet poor plasma was prepared as described above.

Animal models. All animals were housed in a pathogen-free facility with access to food and water ad libitum. Experiments were approved and performed in accordance with the Institutional Animal Care and Use Committee at the University of Texas Southwestern Medical Center.

MDA-MB-231. MDA-MB-231 breast carcinoma cells were maintained in monolayer culture in Eagle's minimum essential medium supplemented with $5 \%$ foetal bovine serum, sodium pyruvate, L-glutamine $(2 \mathrm{mM})$, nonessential amino acids and $2 \times$ vitamin solution. The cultures were incubated at $37^{\circ} \mathrm{C}$ in a humidified atmosphere of $5 \% \mathrm{CO}_{2}$ and $95 \%$ air. Tumour cells were harvested for passage or inoculation by washing the monolayer with PBS, followed by a brief incubation in $0.25 \%$ trypsin/0.02\% EDTA. The cells were collected, washed, resuspended in PBS and confimed $>95 \%$ viable by trypan blue dye exclsuion. For tumour cell implantation, female Balb/c SCID mice were anaesthetised with isoflurane and a 5-mm incision was made in the skin over the lateral thorax. The mammary fat pad was exposed and $0.1 \mathrm{ml}$ of cell suspension $\left(4 \times 10^{7} \mathrm{ml}^{-1}\right)$ was injected into the tissue through a 27-gauge needle. Tumour size was measured with calipers at the indicated time points. Tumour volume was calculated by $D \times d^{2} \times 0.5$, where $D$ is the long diameter and $d$ is the perpendicular short diameter.

MMT-PyMT mice. FVB/N-Tg(MMTV-PyMT)634Mul/J mice were obtained from Jackson Laboratories (Bar Harbor, ME, USA). These transgenic mice express the polyoma virus middle T antigen driven by the MMTV-LTR promoter (Guy et al, 1992). Polioma virus middle $\mathrm{T}$ oncogene expression results in the generation of multifocal mammary carcinomas in $100 \%$ of the mice that is followed by progression to pulmonary metastasis in the majority of animals.

KIC mice. LSL-Kras ${ }^{\mathrm{G} 12 \mathrm{D}}$; Cdkn2a ${ }^{\text {lox/lox}} ; \mathrm{p} 48^{\mathrm{Cre}}$ (mPDAC) mice were generated as previously described (Aguirre et al, 2003; Dineen et al, 2010). Histologically detectable tumour incidence in this genetically engineered model is $100 \%$ at $4-5$ weeks of age and median survival of untreated animals is $\sim 60$ days (Ostapoff et al, 2013).

KPC mice. LSL-Kras ${ }^{\mathrm{G} 12 \mathrm{D} /+}$; LSL-Trp ${ }^{\mathrm{R} 172 \mathrm{H} /+} ; \mathrm{P} 48^{\mathrm{Cre}}$ (KPC) were obtained from National Cancer Institute (NCI) Mouse Repository (Hingorani et al, 2005). In this model, preinvasive lesions become histologically detectable by 10 weeks of age, whereas primary welldifferentiated glandular pancreatic ductal adenocarcima is evident at $\sim 4$ months of age. Median survival of these animals is 5 months and $100 \%$ die by 12 months (Hingorani et al, 2005).

Liposome standards. Large unilamellar vesicles (LUV) were prepared by extrusion through $0.2 \mu \mathrm{m}$ membranes (Avanti miniextruder, Avanti Polar Lipids, Birmingham, AL, USA). Briefly, liposomes were prepared by mixing PS, phosphatidylethanolamine (PE) and phosphatidylcholine (PC) $\left(0.33 \mathrm{mg}\right.$ each) in $\mathrm{CHCl}_{3}$. The lipids were dried under $\mathrm{N}_{2}$, resuspended in $1 \mathrm{ml}$ of HEPES-saline and extruded though the membrane 15 times. Median size of the prepared vesicles was determined by dynamic light scattering and found to be $\sim 171 \mathrm{~nm}$ in diameter (Supplementary Figure S1). For standard curves, we assumed that $50 \%$ of each phospholipid species localised in the outer leaflet of the LUV. Thus $1 \mathrm{mg}$ of LUV containing 33.3\% PS and 33.3\% PE would present with $165 \mu \mathrm{g}$ of PS per $\mathrm{ml}$ and $165 \mu \mathrm{g}$ of PE per $\mathrm{ml}$ accessible for binding and detection, respectively.

ELISA assay. Immunolon $1 \mathrm{~B}$ U-bottomed ELISA plates were coated with $100 \mu \mathrm{l}$ of $\mathrm{KL} 15 \mathrm{C}\left(10 \mu \mathrm{g} \mathrm{ml}^{-1}\right)$ overnight at $4{ }^{\circ} \mathrm{C}$. The plates were then washed with PBS and blocked with BSA (2\% in PBS) at $37^{\circ} \mathrm{C}$ for $1 \mathrm{~h}$. The plates were again washed and the wells were loaded with $100 \mu \mathrm{l}$ of PS/PE/PC (1/1/1) LUV (double diluted from $6 \mu \mathrm{g} \mathrm{ml}^{-1}$ in $50 \%$ normal plasma) or $100 \mu \mathrm{l}$ of a $1 / 2$ dilution 
of test plasma in PBS. The plates were then incubated at $37^{\circ} \mathrm{C}$ for $3 \mathrm{~h}$ and washed with PBS. In all, $100 \mu \mathrm{l}$ of $500 \mathrm{ng} \mathrm{ml}^{-1}$ of biotinylated duramycin (Molecular Targeting Technologies, West Chester, PA. no. B1003) in PBS was added for $1 \mathrm{~h}$ at $20^{\circ} \mathrm{C}$. The plates were then washed with the same buffer and HRPstreptavidin (1/1000 $100 \mu \mathrm{l}$ in PBS) was incubated for $10 \mathrm{~min}$ at $20^{\circ} \mathrm{C}$. After washing with PBS, the plates were developed with $100 \mu \mathrm{l}$ of $\mathrm{OPD}\left(0.5 \mathrm{mg} \mathrm{ml}^{-1}\right)$ and $\mathrm{H}_{2} \mathrm{O}_{2}\left(1 \mu \mathrm{ml}^{-1}\right)$ in $50 \mathrm{~mm}$ citrate phosphate buffer, $\mathrm{pH}$ 4.3. The reaction was stopped with $0.18 \mathrm{M} \mathrm{H}_{2} \mathrm{SO}_{4}$ and absorbance at $490 \mathrm{~nm}$ was determined with a Synergy H1 reader (BioTek Industries, Winoosk, VT, USA).

Statistics. Student's $t$-test was used to evaluate differences in the blood PS levels between tumour-bearing mice and their control littermates. Statistical tests were two sided, and a $P<0.05$ was considered statistically significant. Analysis was performed using SlideWrite Plus V7 (Advanced Graphics Software, Inc., San Diego, CA, USA).

\section{RESULTS}

$\beta 2 \mathrm{GP} 1$ is a plasma protein that is known to bind negatively charged PS at low affinity $(\sim 14 \mu \mathrm{M})$ through a positively charged sequence present in domain 5. Upon dimerisation with $\beta 2 \mathrm{GP} 1$ antibodies however, its affinity to PS increases $>300$-fold (Willems et al, 1996). Because antibody-mediated binding of $\beta 2 \mathrm{GP} 1$ to PSexpressing membranes is also dependent on the affinity of the antibodies to $\beta 2 \mathrm{GP} 1$, we elected to eliminate this dependency by generating an engineered bivalent 'betabody', coined KL15C, consisting of human IgG1 Fc fused to domains 1 and 5 (the PSbinding domain) of $\beta 2 \mathrm{GP} 1$ (Figure 1 and Supplementary Figure S2). In principle, such a bivalent construct will not only have high affinity but will also negate potential assay dependence on $\beta 2 \mathrm{GP} 1$, the amounts of which are highly variable between individuals.

To reduce the possibility of compromised assay sensitivity because of (1) sequestration of exosomal PS to the KL15C capture points that would effectively decrease the density of PS available for binding to (labelled)-annexin 5 and (2) loss of KL15C-captured particles because of competition between the annexin 5 and KL15C, we investigated the use of a PS-independent detection system. Because tumour exosomes contain large amounts of PE (Supplementary Figure S3) and the mole fraction of PE in biological membranes is significantly higher than any membrane protein, we used the PE-specific binding agent, duramycin (Hayashi et al, 1990; Zimmermann et al, 1993), for detection of PS-captured exosomes. Figure 2A shows the high specificity of duramycin and KL15C to PE and PS, respectively. Capture of LUV containing $50 \%$ (wt/wt) PE or PS in PC showed that there was no cross-reactivity between $\mathrm{KL} 15 \mathrm{C}$ and duramycin and duramycin and annexin 5. At high concentrations, however, PE appeared to bind to the KL15C-coated plates. Additional experiments indicated this was due to non-specific binding of PE to BSA used as a blocking agent (Supplementary Figure S4). Taken together, these results suggest that combining KL15C/PS-dependent tumour exosome capture with a duramycin/PE-based detection system would significantly improve assay sensitivity. Figure 2B shows approximately five-fold differential in PS levels between two confirmed ovarian carcinoma patients and two healthy sexmatched individuals using PS capture together with PS detection (KL15C with annexin 5), while using the same plasma, KL15C capture combined with $\mathrm{PE}$ detection (duramycin) increased sensitivity $>25$-fold ( $>140$-fold difference between malignant and healthy). Interestingly, an identical experiment using duramycin-dependent PE capture in lieu of KL15C-dependent PS capture resulted in a dramatic loss in sensitivity (Figure $2 \mathrm{C}$ ). This was likely due to a significant decrease in assay stringency because,

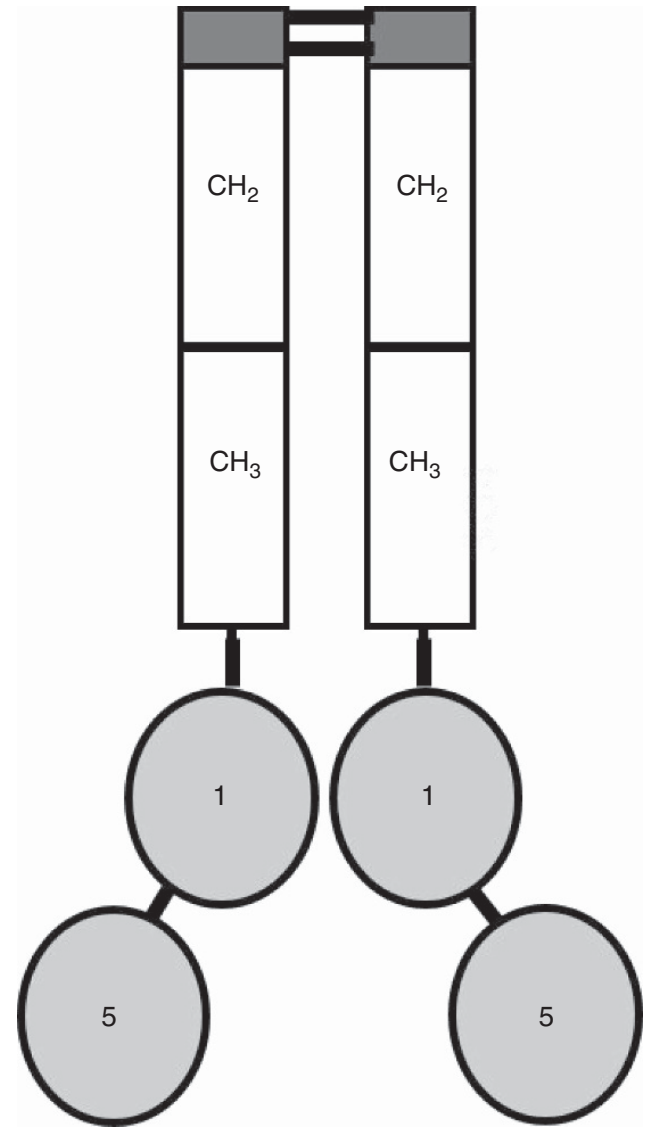

Figure 1. Schematic diagram of betabody, KL15C. Domains 1 and 5 of the plasma PS-binding protein, $\beta 2 \mathrm{GP} 1$, were genetically fused to the C-terminus of the $\mathrm{CH} 3$ domains of the $\mathrm{Fc}$ fragment of human IgG1.

A Gly4Ser linker was inserted between the $\mathrm{CH} 3$ domains and domains 1 and 5 of $\beta 2 \mathrm{GP} 1$. The recombinant betabody was expressed as a dimer.

while KL15C captures only PS-expressing tumour exosomes, duramycin captures all exosomes of which only a fraction are tumour exosomes. As previously suggested (Logozzi et al, 2009; Cappello et al, 2017), these data indicate that cancer patient blood contains significantly more exosomes than blood from normal individuals. Nonetheless, the presence of a malignancy cannot be confirmed in the absence of a tumour-specific biomarker such as PS. Taken together, these data show that an ELISA employing PSdependent capture with PE-dependent detection significantly improves assay sensitivity without compromising system specificity.

Animal studies. Several mouse model systems were employed to determine the minimal tumour burden required for detection of PS-expressing tumour-derived exosomes in blood. These included a transplantable xenogeneic human breast cancer model (MDAMB-231) and three genetic models, breast (PyMT) and pancreatic (KIC and KPC) cancers, that produce spontaneous tumours at 2-5 months of age.

$M D A-M B 231$. Five 8-week-old SCID mice were injected orthotopically with $4 \times 10^{6}$ cells. The animals were bled just before tumour implantation and weekly thereafter. Plasma was pooled and stored at $-20^{\circ} \mathrm{C}$ until assayed. Although the smallest palpable tumours were first observed at day 28, increased blood PS levels were already detected at day 7 and continued to increase with tumour size (Figure 3A). Assessment of blood exosome numbers did not reveal measurable increases until day 14 (Figure 3B), 7 days later than tumour could be detected by PS ELISA (Figure 3A). It should be 

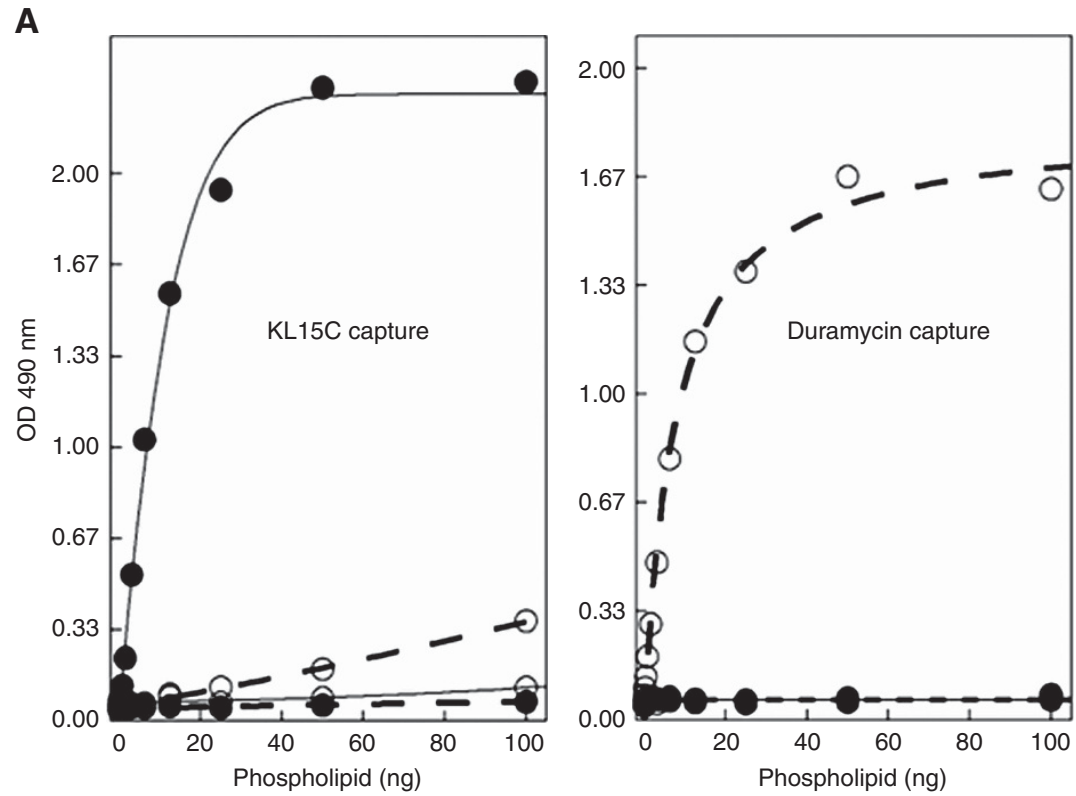

B

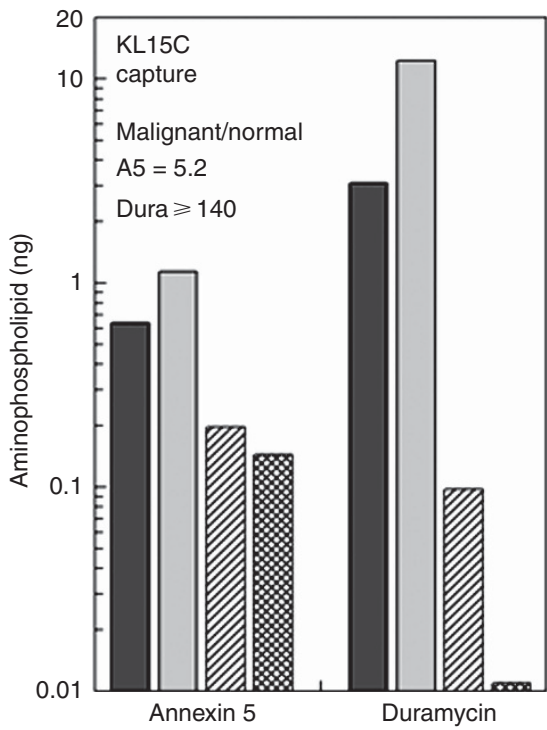

C

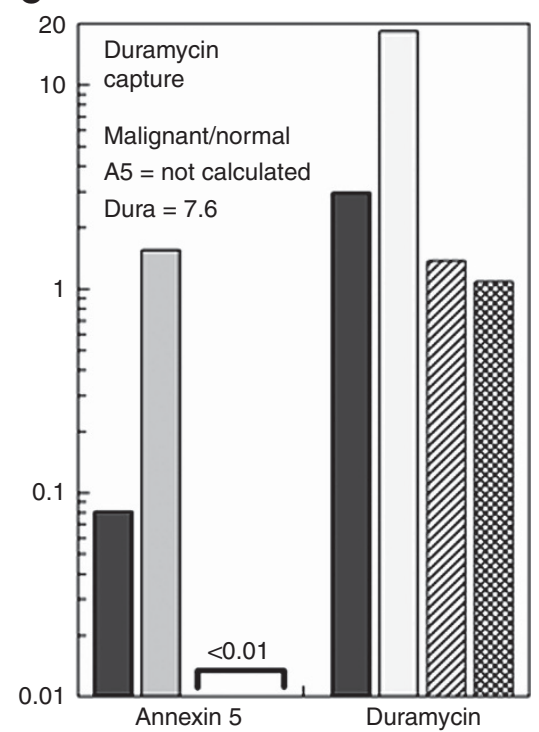

Figure 2. Determination of blood exosomal PS levels for the detection of tumours. (A) ELISA plates coated with KL15C (PS capture) and duramycin (PE capture) were incubated with the indicated amounts of vesicles containing $50 \%$ PS or 50\% PE (wt/wt) in PC. After washing, the plates were incubated with biotinylated annexin 5 in the presence of $\mathrm{Ca}^{2+}(2 \mathrm{~mm})$ or biotinylated duramycin (no $\mathrm{Ca}^{2+}$ ) and the amounts of PS or PE captured was quantified with HRP-streptavidin. The amounts of phospholipid indicated on the abscissa represents the assumed amounts of aminophospholipids expressed on the vesicle surface that is available for binding ( $50 \%$ aminophospholipid in PC with half of that in the particles outer leaflet). Closed circles, PS/PC vesicles; open circles, PE/PC vesicles; solid line, annexin 5 detection (PS); dotted line, duramycin detection (PE). (B and C) Human EDTA plasma (100 $\mu \mathrm{l}$ of 50\% diluted plasma in PBS) from two confirmed ovarian carcinoma patients (dark grey and light grey) or two sex-matched healthy individuals (diagonal lines and crossed lines) were incubated on ELISA plates coated with (B) KL15C or (C) duramycin. After exosome capture, the plates were developed with both probes as described for the synthetic vesicles in (A). The values shown in (B and C) were calculated from the standard PS and PE curves shown in (A) (PS, left; PE, right) and are the average of two confirmed cancer patients and two healthy individuals.

noted that while there was a direct relationship between blood PS levels and the number of exosomes through day 28, the ratio of PE/ exosome decreased after day 28 (Figure $3 \mathrm{~B}$ ). This result is consistent with the apparent saturation of detectable PE shown in Figure 3A.

MMTV-PyMT. Five PyMT transgenic mice and four littermate controls were bled beginning at day 35 of age and weekly thereafter and monitored for tumour growth. Figure 3C shows that blood tumour exosome levels seemed to increase at day $\sim 42$ although the differences between the two groups were not statistically significant. By day 49, however, statistically significant differences were observed $(P<0.0002$; Figure $3 \mathrm{D})$. Palpable tumours were observed only after $\sim 60$ days. No tumours were detected in the littermate control animals. Values from individual mice and their littermate controls are shown in Supplementary Figure S5.

KIC. Fourteen KIC transgenic mice and 14 littermate controls were bled beginning at day 21 of age and weekly thereafter. Mice were killed when their body weights decreased $>1 \mathrm{~g}$ from the previous week's value and the weights of the pancreas were 
determined (Supplementary Table S1). Figure 3E shows that the average blood tumour exosome levels were already significantly higher than the control group at the first bleed on day 21 $(P<0.001$; Figure 3F). Tumour-positive sonograms, however, could only be obtained after day 40 (Supplementary Figure S6B).
In all, $100 \%$ of the KIC mice developed pancreatic tumours (see one exception, Supplementary Table S1). No tumours were observed in the littermate controls (Supplementary Figure S6C). Values from individual mice and their littermate controls are shown in Supplementary Figure S6A.
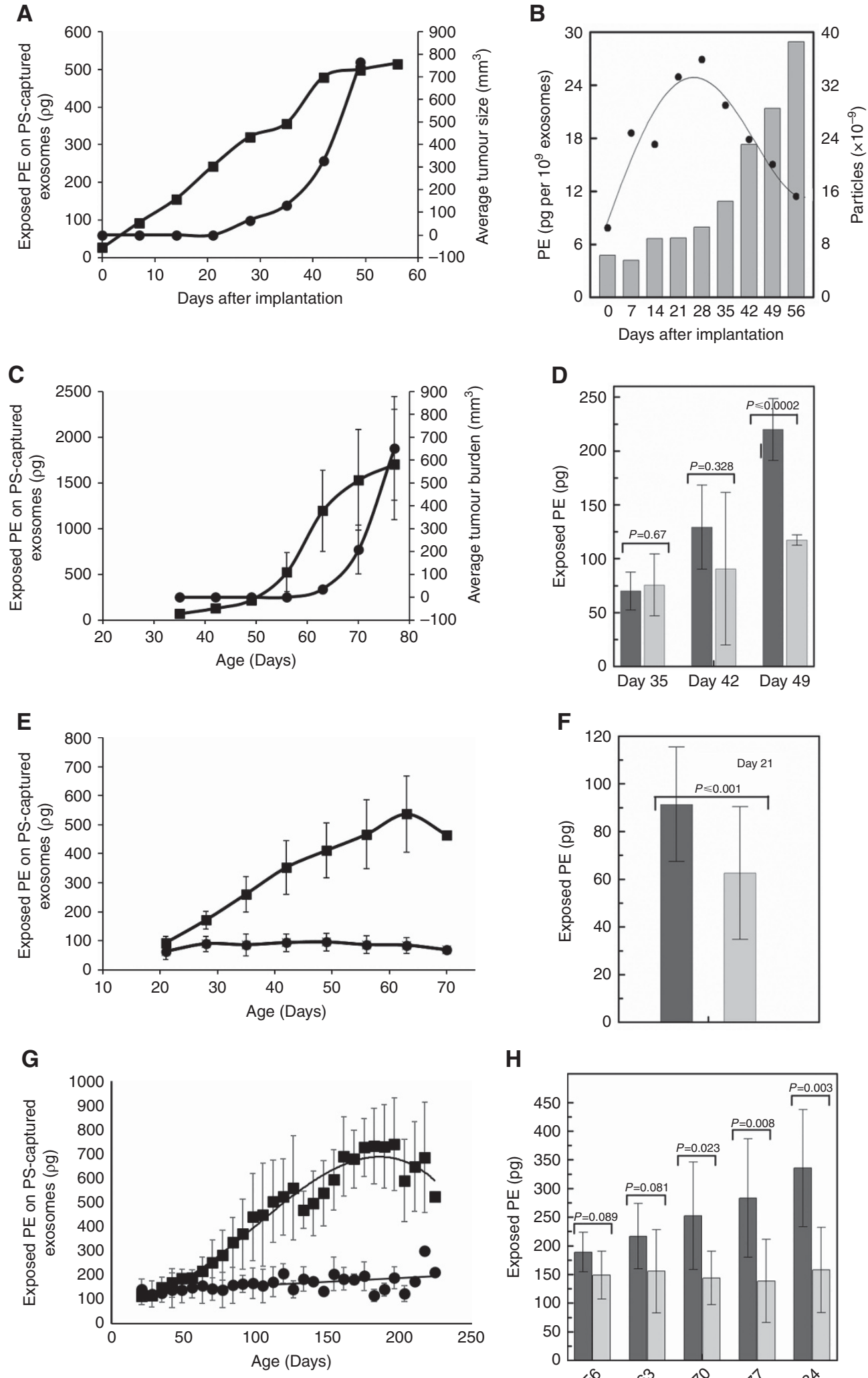
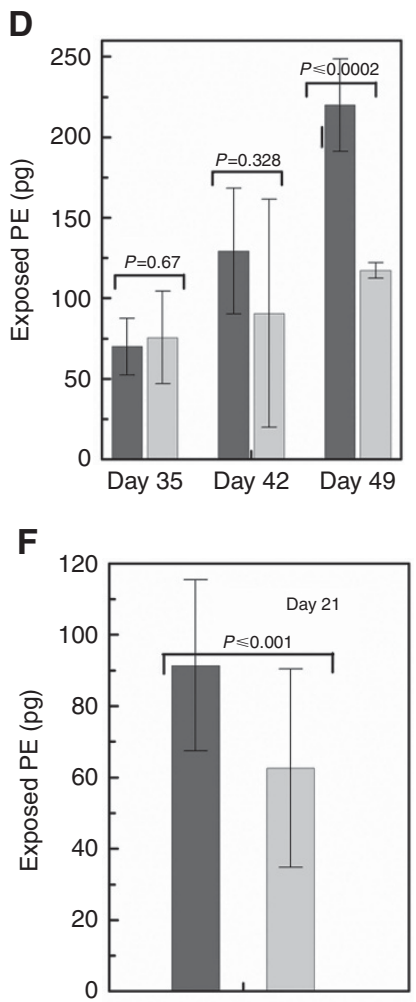

H

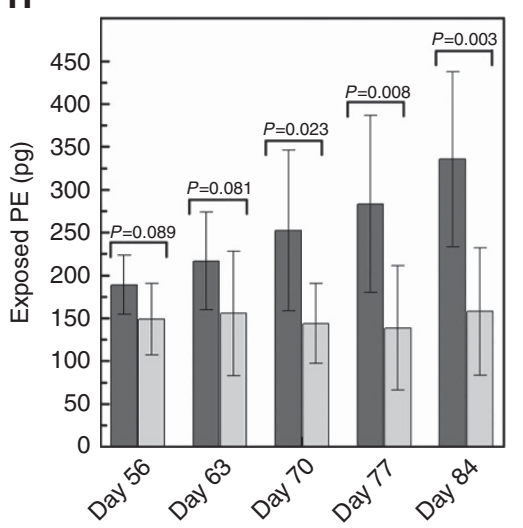

Figure 3. For caption see next page. 
KPC. Nine KPC transgenic mice and eight littermate controls were bled beginning at 21 days of age and weekly thereafter. Mice were killed when their body weights decreased $>1 \mathrm{~g}$ from the previous weeks' value and the weights of the pancreas were determined (Supplementary Table S2). Figure 3G shows that the average tumour exosome levels began to increase by day 35 and was significantly higher $(P=0.023)$ than the littermate controls by day 70 (Figure $3 \mathrm{H}$ ).

\section{DISCUSSION}

Analysis of blood exosomes for cancer biomarkers has recently come to the forefront of liquid biopsy-based cancer diagnostics. For the most part, these studies are based on increased blood exosome levels (Logozzi et al, 2009; Cappello et al, 2017) and the isolation (Melo et al, 2015; De Palma et al, 2016; Dong et al, 2016; Welton et al, 2016) or capture (Jorgensen et al, 2013; Jakobsen et al, 2015) of plasma exosomes, which are then probed for specific RNA (De Palma et al, 2016; Dong et al, 2016; Fais et al, 2016) or protein signatures (Jakobsen et al, 2015; Melo et al, 2015; Fais et al, 2016; Welton et al, 2016). Although specific biomarkers can be diagnostic for a certain tumour type, a surrogate pan-cancer biomarker could find utility in the diagnosis of many cancer types. This is particularly relevant for cancers for which there are no routine screening protocols (e.g., renal, brain, bone and pancreatic cancer). Moreover, such a biomarker could be predictive of recurrence and response to therapy.

In this study, we describe an ELISA-based assay system that detects picogram amounts of phospholipid exposed on exosomes in the blood of tumour-bearing mice. Unlike previously described protein-dependent exosome capture and detection ELISAs (Logozzi et al, 2009), the detection system described here is based on highly PS-specific $\beta 2 \mathrm{GP} 1$-mediated exosome capture and quantitation of exosomal PE with duramycin. This system overcomes potential deficiencies in our recently described assay system (Lea et $a l, 2017$ ) where quantitation could have been compromised because of varying $\beta 2 \mathrm{GP} 1$ levels between individuals and competition between the binding (capture) of $\beta 2 \mathrm{GP} 1$ to PS and exosome detection with annexin 5 that also binds PS. Moreover, as $\mathrm{Ca}^{2+}$ is known to inhibit $\beta 2 \mathrm{GP} 1$ binding to PS (Willems et al, 1996) but is required for annexin 5 detection of PS, the current $\mathrm{Ca}^{2+}$-free system eliminates any potential errors that might have occurred due to competition between annexin 5 and $\beta 2 \mathrm{GP} 1$ that could have led to release of captured exosomes from the plastic plate.
In both breast cancer models, PS-expressing exosomes were detected long before the tumours became palpable. In the MDAMB-231 and genetic MMTV-PyMT models, detectable increases in tumour exosome blood levels were seen at day 7 after tumour cell inoculation and 42 days of age, while palpable tumours were observed only after days 28 and 63, respectively. Consistent with elevated blood exosome concentrations in cancer patients, the data presented in Figures $3 \mathrm{~A}$ and $\mathrm{B}$ show a concomitant increase in both tumour size and exosome number. In addition, the data show a dramatic increase in the amount of PE/particle through day 28. With continued tumour growth, however, the ratio of $\mathrm{PE}$ to particles decreased (Figure 3B). Although this still needs to be confirmed, this was likely due to the saturation of the PE curve at large tumour volumes after day 40 (Figure $3 \mathrm{~A}$ ). In principle, this could be due to saturation of PS-dependent capture and/or PEdependent detection.

Blood tumour exosome levels were significantly elevated in the pancreatic cancer KIC mice at 21 days of age and in the KPC mice at 70 days of age when only low-grade preinvasive lesions can be seen (Aguirre et al, 2003; Hingorani et al, 2005). For the KIC model, histologically confirmed high-grade adenocarcinomas generally appear at 35 days of age (Aguirre et al, 2003) where blood exosome levels were three-fold higher than the levels of age-matched littermate controls (260 vs $85 \mathrm{pg}$ ). The first visible lesions on sonograms appear only after day 40 (Supplementary Figure S6B) where PS exosome levels increase to $>350 \mathrm{pg}$. For the KPC model, histologically confirmed preinvasive pancreatic intraepithelial neoplasia (PanIn) lesions generally appear at 10 weeks of age (Hingorani et al, 2005) where blood exosome levels were $\sim 1.8$-fold higher than the levels of age-matched littermate controls (252 vs $143 \mathrm{pg}$ ). The first histologically confirmed adenocarcinomas appear at $\sim 4$ months of age (Hingorani et al, 2005) when the tumour exosome levels in the KPC mice are $\sim 4$-fold higher than in the litter controls (560 vs 142 pg).

In summary, this study provides data that support the high diagnostic power of quantifying PS-expressing tumour exosomes in blood. Although it is difficult to extrapolate these results to tumours in humans, the ability to detect PS-expressing tumour exosomes in the blood of animals earlier than any clinical manifestations of disease suggests that this assay system could find utility in the early detection of human cancers. It should be noted that the assay cannot distinguish between different tumour types or their tissue of origin. From a clinical perspective, this may be viewed as a significant limitation; however, if it indeed diagnoses early disease, further patient workup could determine disease site. Importantly, the test could be useful in population screens by

Figure 3. Detection of PS-expressing tumour-derived exosomes in the blood of tumour-bearing mice. (A) MDA-MB231 breast cancer model: 8-week-old SCID Balb/c mice were injected orthotopically with $4 \times 10^{6}$ MDA-MB231 cells on day 0 . Day 0 blood was taken just before inoculation with tumour cells. The mice were then bled at weekly intervals. Each data point represent $50 \mu$ l of pooled plasma from 5 mice. Squares, exposed PE levels on PS-captured exosomes; circles, average tumour volume of the 5 mice. (B) Blood exosome numbers in pooled plasma of mice bearing MDA-MB231 tumours (bars): Aliquots of plasma obtained from animals described in (A) were quantified for total exosome number using the Exocet Exosome Quantification Assay system as described by the manufacturer (Systems Biosciences Inc., Palo Alto, CA, USA). The ratio of PS to particle number (circles) was obtained by dividing the PE values in (A) by the particle count. (C) PYMT breast cancer model: Five FVB PyMT mice were monitored for tumour size and bled weekly for exosome quantification beginning at day 35. Squares, exposed PE levels on PS-captured exosomes; circles, tumour volume. The data represent the average and s.d. for all animals. (D) Student's t-test $P$-values for days 35,42 and 49 . Dark grey, PYMT mice; light grey, littermate controls. Individual tumour and exosome plots are shown in Supplementary Figure S5. (E) KIC pancreatic cancer model: Fourteen KIC mice and 14 littermate controls were monitored for body weight and bled weekly for exosome quantification beginning at day 21. Squares, exposed PE levels on PS-captured exosomes; circles, exposed PE levels in littermate control animals. The data represent the average and s.d. for all animals. (F) Student's t-test $P$-values for day 21. Dark grey, KIC mice; light grey, KIC control littermates. Individual exosome plots are shown in Supplementary Figure S6A. (G) KPC pancreatic cancer model: Nine KPC mice and 8 littermate controls were monitored for body weight and bled weekly for exosome quantification beginning at day 21. Squares, exposed PE levels on PS-captured exosomes; circles, exposed PE levels in littermate control animals. The data represent the average and s.d. for all animals. (H) Student's t-test $P$ values for days 56-84. Dark grey, KPC mice; light grey, KPC control littermates. Individual data sets are shown in Supplementary Figure S7. 
detecting indolent asymptomatic disease that would lead to earlierstage diagnosis resulting in improved clinical outcomes.

\section{ACKNOWLEDGEMENTS}

The research was funded by Cancer Prevention and Research Institute of TX (CPRIT) Grant no. RP110441 with additional support from Simmons Cancer Center Support Grant 5P30 CA142543. We thank Jason Toombs and Tara Billman for the sonogram data, animal bleeds and necropsy and Dr Rajiv Nayar from HTD Biosystems, Pleasanton, CA for the dynamic light scattering analysis.

\section{CONFLICT OF INTEREST}

The authors declare no conflict of interest.

\section{AUTHOR CONTRIBUTIONS}

RS carried out experiments; AJS and RS did the statistical analyses and interpretation of data; AJS, $\mathrm{XH}$ and RAB provided administrative and material support; AJS and RS conceived and designed the study; AJS and RS wrote the manuscript. All authors read and approved the final manuscript.

\section{REFERENCES}

Aguirre AJ, Bardeesy N, Sinha M, Lopez L, Tuveson DA, Horner J, Redston MS, DePinho RA (2003) Activated Kras and Ink4a/Arf deficiency cooperate to produce metastatic pancreatic ductal adenocarcinoma. Genes Dev 17: 3112-3126.

Bujak E, Pretto F, Neri D (2015) Generation and tumor recognition properties of two human monoclonal antibodies specific to cell surface anionic phospholipids. Invest New Drugs 33: 791-800.

Cappello F, Logozzi M, Campanella C, Bavisotto CC, Marcilla A, Properzi F, Fais S (2017) Exosome levels in human body fluids: a tumor marker by themselves? Eur J Pharm Sci 96: 93-98.

Connor J, Bucana C, Fidler IJ, Schroit AJ (1989) Differentiation-dependent expression of phosphatidylserine in mammalian plasma membranes: quantitative assessment of outer-leaflet lipid by prothrombinase complex formation. Proc Natl Acad Sci USA 86: 3184-3188.

De Palma G, Sallustio F, Curci C, Galleggiante V, Rutigliano M, Serino G, Ditonno P, Battaglia M, Schena FP (2016) The three-gene signature in urinary extracellular vesicles from patients with clear cell renal cell carcinoma. J Cancer 7: 1960-1967.

Dineen SP, Roland CL, Greer R, Carbon JG, Toombs JE, Gupta P, Bardeesy N, Sun H, Williams N, Minna JD, Brekken RA (2010) Smac mimetic increases chemotherapy response and improves survival in mice with pancreatic cancer. Cancer Res 70: 2852-2861.

Dong HP, Holth A, Kleinberg L, Ruud MG, Elstrand MB, Trope CG, Davidson B, Risberg B (2009) Evaluation of cell surface expression of phosphatidylserine in ovarian carcinoma effusions using the annexinV/7-AAD assay: clinical relevance and comparison with other apoptosis parameters. Am J Clin Pathol 132: 756-762.

Dong L, Lin W, Qi P, Xu MD, Wu X, Ni S, Huang D, Weng WW, Tan C, Sheng W, Zhou X, Du X (2016) Circulating long RNAs in serum extracellular vesicles: their characterization and potential application as biomarkers for diagnosis of colorectal cancer. Cancer Epidemiol Biomarkers Prev 25: 1158-1166.

Fais S, O'Driscoll L, Borras FE, Buzas E, Camussi G, Cappello F, Carvalho J, Cordeiro da Silva A, Del Portillo H, El Andaloussi S, Ficko Trcek T, Furlan R, Hendrix A, Gursel I, Kralj-Iglic V, Kaeffer B, Kosanovic M, Lekka ME, Lipps G, Logozzi M, Marcilla A, Sammar M, Llorente A, Nazarenko I, Oliveira C, Pocsfalvi G, Rajendran L, Raposo G, Rohde E, Siljander P, van Niel G, Vasconcelos MH, Yanez-Mo M, Yliperttula ML, Zarovni N, Zavec AB, Giebel B (2016) Evidence-based clinical use of nanoscale extracellular vesicles in nanomedicine. ACS Nano 10: 3886-3899.

Golde WT, Gollobin P, Rodriguez LL (2005) A rapid, simple, and humane method for submandibular bleeding of mice using a lancet. Lab Anim (NY) 34: 39-43.

Guy CT, Cardiff RD, Muller WJ (1992) Induction of mammary tumors by expression of polyomavirus middle $\mathrm{T}$ oncogene: a transgenic mouse model for metastatic disease. Mol Cell Biol 12: 954-961.

Hayashi F, Nagashima K, Terui Y, Kawamura Y, Matsumoto K, Itazaki H (1990) The structure of PA48009: the revised structure of duramycin. J Antibiot (Tokyo) 43: 1421-1430.

Hingorani SR, Wang L, Multani AS, Combs C, Deramaudt TB, Hruban RH, Rustgi AK, Chang S, Tuveson DA (2005) Trp53R172H and KrasG12D cooperate to promote chromosomal instability and widely metastatic pancreatic ductal adenocarcinoma in mice. Cancer Cell 7: 469-483.

Jakobsen KR, Paulsen BS, Baek R, Varming K, Sorensen BS, Jorgensen MM (2015) Exosomal proteins as potential diagnostic markers in advanced non-small cell lung carcinoma. J Extracell Vesicles 4: 26659.

Jorgensen M, Baek R, Pedersen S, Sondergaard EK, Kristensen SR, Varming K (2013) Extracellular vesicle (EV) array: microarray capturing of exosomes and other extracellular vesicles for multiplexed phenotyping. $J$ Extracell Vesicles 2: 20920.

Keller S, Konig AK, Marme F, Runz S, Wolterink S, Koensgen D, Mustea A, Sehouli J, Altevogt P (2009) Systemic presence and tumor-growth promoting effect of ovarian carcinoma released exosomes. Cancer Lett 278: 73-81.

Khan S, Jutzy JM, Valenzuela MM, Turay D, Aspe JR, Ashok A, Mirshahidi S, Mercola D, Lilly MB, Wall NR (2012) Plasma-derived exosomal survivin, a plausible biomarker for early detection of prostate cancer. PLoS One 7: e46737.

Lea J, Sharma R, Yang F, Zhu H, Ward ES, Schroit AJ (2017) Detection of phosphatidylserine-positive exosomes as a diagnostic marker for ovarian malignancies: a proof of concept study. Oncotarget 8: 14395-14407.

Logozzi M, De Milito A, Lugini L, Borghi M, Calabro L, Spada M, Perdicchio M, Marino ML, Federici C, Iessi E, Brambilla D, Venturi G, Lozupone F, Santinami M, Huber V, Maio M, Rivoltini L, Fais S (2009) High levels of exosomes expressing CD63 and caveolin-1 in plasma of melanoma patients. PLoS One 4: e5219.

Melo SA, Luecke LB, Kahlert C, Fernandez AF, Gammon ST, Kaye J, LeBleu VS, Mittendorf EA, Weitz J, Rahbari N, Reissfelder C, Pilarsky C, Fraga MF, Piwnica-Worms D, Kalluri R (2015) Glypican-1 identifies cancer exosomes and detects early pancreatic cancer. Nature 523(7559): 177-182.

Noerholm M, Balaj L, Limperg T, Salehi A, Zhu LD, Hochberg FH, Breakefield XO, Carter BS, Skog J (2012) RNA expression patterns in serum microvesicles from patients with glioblastoma multiforme and controls. BMC Cancer 12: 22.

Ostapoff KT, Awasthi N, Cenik BK, Hinz S, Dredge K, Schwarz RE, Brekken RA (2013) PG545, an angiogenesis and heparanase inhibitor, reduces primary tumor growth and metastasis in experimental pancreatic cancer. Mol Cancer Ther 12(7): 1190-1201.

Rabinowits G, Gercel-Taylor C, Day JM, Taylor DD, Kloecker GH (2009) Exosomal microRNA: a diagnostic marker for lung cancer. Clin Lung Cancer 10: 42-46.

Riedl S, Rinner B, Asslaber M, Schaider H, Walzer S, Novak A, Lohner K, Zweytick D (2011) In search of a novel target-phosphatidylserine exposed by non-apoptotic tumor cells and metastases of malignancies with poor treatment efficacy. Biochim Biophys Acta 1808: 2638-2645.

Szajnik M, Derbis M, Lach M, Patalas P, Michalak M, Drzewiecka H, Szpurek D, Nowakowski A, Spaczynski M, Baranowski W, Whiteside TL (2013) Exosomes in plasma of patients with ovarian carcinoma: potential biomarkers of tumor progression and response to therapy. Gynecol Obstet (Sunnyvale) Suppl 4: 3.

Utsugi T, Schroit AJ, Connor J, Bucana CD, Fidler IJ (1991) Elevated expression of phosphatidylserine in the outer membrane leaflet of human tumor cells and recognition by activated human blood monocytes. Cancer Res 51: 3062-3066.

Vallabhapurapu SD, Blanco VM, Sulaiman MK, Vallabhapurapu SL, Chu Z, Franco RS, Qi X (2015) Variation in human cancer cell external phosphatidylserine is regulated by flippase activity and intracellular calcium. Oncotarget 6: 34375-34388.

Welton JL, Brennan P, Gurney M, Webber JP, Spary LK, Carton DG, Falcon-Perez JM, Walton SP, Mason MD, Tabi Z, Clayton A (2016) 
Proteomics analysis of vesicles isolated from plasma and urine of prostate cancer patients using a multiplex, aptamer-based protein array. J Extracell Vesicles 5: 31209.

Willems GM, Janssen MP, Pelsers MM, Comfurius P, Galli, Zwaal RF,

Bevers EM (1996) Role of divalency in the high-affinity binding of anticardiolipin antibody-beta 2-glycoprotein I complexes to lipid membranes. Biochemistry 35: 13833-13842.

Wurm H (1984) beta 2-Glycoprotein-I (apolipoprotein H) interactions with phospholipid vesicles. Int J Biochem 16: 511-515.
Zimmermann N, Freund S, Fredenhagen A, Jung G (1993) Solution structures of the lantibiotics duramycin B and C. Eur J Biochem 216: 419-428.

This work is published under the standard license to publish agreement. After 12 months the work will become freely available and the license terms will switch to a Creative Commons AttributionNonCommercial-Share Alike 4.0 Unported License.

Supplementary Information accompanies this paper on British Journal of Cancer website (http://www.nature.com/bjc) 